\title{
Sindrome de San Filippo: tipificación de mucopolisacáridos en orina y determinación enzimática en plasma.
}

Drs. Raquei Burrows* , Santiago Muzzo B.*

\begin{abstract}
Three cases of Sanfilipm s Syndrome or Mucopolysaceharidues Type III are presented. Typificalion of mucupolysaccharides in urine revealed increased urinary Heparan Sulfuce excretion and determination of enzyme activities in serum demogtrated normal Alfa-Hexcsaminidase activities, roulling out Sanfilippo Syndrome Type B. Fibroblast tozyme artivities measurement are necessary tn centify type A or C. The importance of diagnusing accurately with these laboratory exams the Iype of Muenopoliaccharidosis, is discused. Special emphusis is given to the analygis of the prognasis and treatment of sanfilippo's Symotrome.
\end{abstract}

Las mucopolisacaridosis son un grupo heterogéneo de enfermedades de depósito caracterizadas por una alteración congénita de las enzimas que partjcipan en el metabolismo de los mucopolisacáridos (MPS). En ellas existe un acúmulo de MPS en los diferentes parénquimas, los que producen el cuadro clinico. bioquimico y radiológico de estos pacientes.., 2

En 191 ? Hunter y Hurler describieron pacientes con facie peculiar, retardo mental y alteraciones íseas. Por muchos años se clasificaron a todos estos pacientes como Hurler a Hunter, recibiendo en general la denominarión de gargoilismo, por la facie similar a una gárgola. La identificación de los MPS permitió conocer que estas enfermedades tenían en común una excreción aumentada de MPS en la orina 3. \& y el estudio del tipo de herencia reveló que eran autosómicas recesivas y sólo el Hunter ligado al sexo.

En los años 60, con el aislamiento de los diferentes tipos de MPS, se vio que en estas enfermedades existian varios modelos de excreción urinaria de MPS 5. 6

En 1966. McKusick clasificó las mucopolísacaridosis de acuerdo al cuadro clínico, tipo de herencia y modelo de excreción de MPS en orina, describiendo 6 gapos: Tipo I o Hurler, Tipo II o Hunter, Tipo

* Serviciós de Pediatría Hospítal Sátero del Rio y Unidad de Endor crinolozia del Instituto de Nutrición y Tecnología de los Alitnanlos. Universidad de Chile, Santiago, Chile.
III o Sanfilippo. Tipo IV o Morquio. Tipo Vo Scheie y Tipo VI o Maroteaux Lamy. ${ }^{\text {? }}$

El estudio ultraestructural y la unión de sulfato radiactivo a los MPS de fibroblastos de estos pacientes demostró acúmulo intralisosomal de estos compuestos. ${ }^{21 .} 12$

Fue posible conocer los distintos tipos de mucopolisacaridosis, al observar en fibroblastos de piel de pacientes desaparición del acúmulo patológico de MPS al cultivar estas células con otras de sujetos normales o afectados por otro tipo de mucopolisacaridosis y persistencia de este acúmulo, al cultivarlos con los de pacientes con el mismo déficit enzimático. ${ }^{13 .}{ }^{14}$ Estos experimentos sugirieron diferentes enzimas deficitarias en cada tipo de Mucopolisacaridosis. La medición enzimática direc$\mathrm{ta}^{\text {}}{ }^{\text {to }}$ permite conocer en forma exacta la enzima en déficit y detectar heterocigotos con déficit enzimático parcial. Las mediciones enzimáticas pueden ser hechas en fibroblastos para todas las Mucopolisacaridosís y en plasma o suero sólo para algunas, pues por ser enzimas intralisosomales se encuentran generalmente dentro de las células, existiendo cantidades minimas fuera de ellas. Surgió entonces una nueva clasificación de la original de YcKusick en e] que se considera el tipo de enzima en déficit. ${ }^{2}$ Tabla 1 .

El Síndrome de Sanfilippo o Mucopolisacaridosis tipo III, puede presentarse con alteraciones somáticas severas similares al Hurler o Hunier, o 


\section{MUCOPOLISACARIDOSIS}

NOMBRE EN EXCESO

- Tipo I ó Hurler Opacidad corneal, PMl, ALTERAC IONES OSEAS, MUERTE TEMPRANA

- Tipo IS ó Scheie Rigidez articular o OPACIDAD CORNEAL, INSUFICIENCIA AORTICA, CI NORMAL

- Tipo ihS ó Hurler fenotipo intermedio SCHEIE

- Tipo IIA ó Hunter Sin opacidad corneal. SEVERo MENOS gRAVES QUE EL TIPO IH MUERTE TEMPRANA.LIGADO AL SEXO

- Tipo lib ó hunter Sobrevida hasta los Mediano 30-50 ANOS, CI NORMAL

- Tipo III a ó SAMFILIPPO A

- TIPO III B ó SANFILIPPO B

- TIPO III C ó SANFILIPPO C

- TIPO IV O MORQuio

RH SEVERO, SIN OPACIDAD CORNEAL, ALTERACIONES ośEAs LEYES.

PM MENOS SEVERo

RY SEVERO, CONTRACTURA ARTICULAR

ALteraciones OSEAS SEVERAS, RM Y OPAC IDAD CORMEAL AUSENTE O TARDIOS

- Tipo V: vacante

- Tipo Vi a o maro- alteraciones oseas y teaux Lamy clasi- corneales severas, CI CO NORMAL

- Tipo VI B ó MaroTEAUX LAMY LEYE

- TIPO VII ó deF JCIT GLUCURONIDA$S A$
Dermatan y Heparan SULFATO

Dermatan y Heparan SULFATO

Dermatan y Heparan SULFATO

Dermatan y Heparan Sulfato

$\alpha-1$-IDUROMIDASA

$\prec$-1-IDURONIDASA

SULFOIDURONIDASA SULFATASA

Dermatan y Heparan SULFATO

Heparan Sulfato

Heparaam Sulfato

Heparam Sulfato

KERATAN SULFATO

Dermatan Sulfato

Bermatan Sulfato

Dermatan y hieparan SULFATO
Heparan sulfato SULFATASA

$\propto N$-ACETIL-D- GLUCOSAMINIDASA

$\propto$ GLUCOSAMIMIDASA- $N$ ACETIL TRANSFERASA

N-ACET ILHEXOSAMINIDASA 4-\$04 SLLFATASA

N-ACETILHEXOSAMIN IDASA 6-SO4 SULFATASA

-GLUCURONIDASA 
simplemente como un retardo mental de etiología no precisada. El diagnóstico suele hacerse después de los tres años de edad. Las primeras alteraciones suelen verse en el desarrollo psicomotor, sin que hasta entonces aparezcan manifestaciones somáticas. Este retraso es progresivo comenzando con una hiperactividad, conducta agresiva y alteraciones del sueño, llegando a veces a retardos mentales Beveros con pérdida del lenguaje y del control de esfinteres. El aspecto facial suele ser poco tosco con escaso compromiso pondoestatural y cardíaco. La opacidad corneal de existir es tardía, pero el compromiso de retina suele ser precoz, describiéndose una renitis pigmentaria. Con frecuencia hay rigidez articular. Radiológicamente presentan cuerpos vertebrales ovoideos, costillas espatuladas, aplanamiento del techo acetabular y engrosamientos óseos. El hallazgo de células de Buhot en médula ósea es típico en este síndrome. Existe depósito laminar concéntrico de MPS en neuronas de la corteza a diferencia de otras mucopolisacaridosis donde el depósito es granular. En el síndrome de Sanfilippo sólo existe dificultad en la degradación de Heparan Sulfato, siendo el único MPS que se encuentra aumentado en orína.

Actualmente hay descritos 3 tipos de Sanfilippo clínicamente indistinguibles, pero diferentes en cuanto a la enzima en déficit. llamados Sanfilippo $A$, B y C. en los que existen déficit de Heparan- $\mathrm{N}$-sulfatasa. N-cetil alfa Glucosaminidasa y alfaglucosaminidasa-N-cetil-transferasa, respectivamente ${ }^{k}{ }^{\text {S }} \mathbf{L a}$ deficiencia enzimática en el Sanfilippo A puede ser detectada en fibroblastos o leucocitos, en el tipo $B$ se puede medir además en plasma y suero y en el tipo $\mathrm{C}$, sólo en fibroblastos. ${ }^{10}$

Se presentan en esta oportunidad 3 casos clínicos de Sanfilippo en los cuales se efectuó tipificación de MPS en orina y determinación enzimática sérica.

Caso clínico I: A. M. R., paciente de 4 años, de sexo femenino. producto de padres jóvenes no consanguíneos. Embarazo, parto y período de recién nacido normales. 3 hermanos mayores sanos. Bronquitis y diarreas a repetición desde los primeros meses de vida, que produjeron una desnutrición de II grado en el primer año de vida. Retraso severo de talla (bajo el percentil 3) y del desarrollo psicomotor (edad mental de 2 años), macrocefalia relativa, pelo grueso y abundante, hipertricosis, facie tosca con cejas hiperpobladas, sinofrísis, labios gruesos, macroglosia y alteraciones dentarias. Tórax simétrico con estertores pulmonares abundantes. Abdomen globulosu con cicatriz de herniorrafia umbilical e hígado a 4 cm bajo el reborde costal. Dedos de manos y pies cortos y gruegos. Sin opacidad ni alteraciones de retina. E] examen radiológico reveló una silla turca normal, cuerpos vertebrales redondeados (Figura 1). costillas espatuladas y retrago marcado de edad ósea (1 3/12 años para 3 8/12 años de edad cronológica). Screening de MPS en orina positivo. La tipificación de MPS en orina reveló exceso de heparan sulfato $y$ la determinación de actjvidad enzimática plasmática de Alfahexosaminidasas fue normal, lo que descartó un Sanfilippo B.

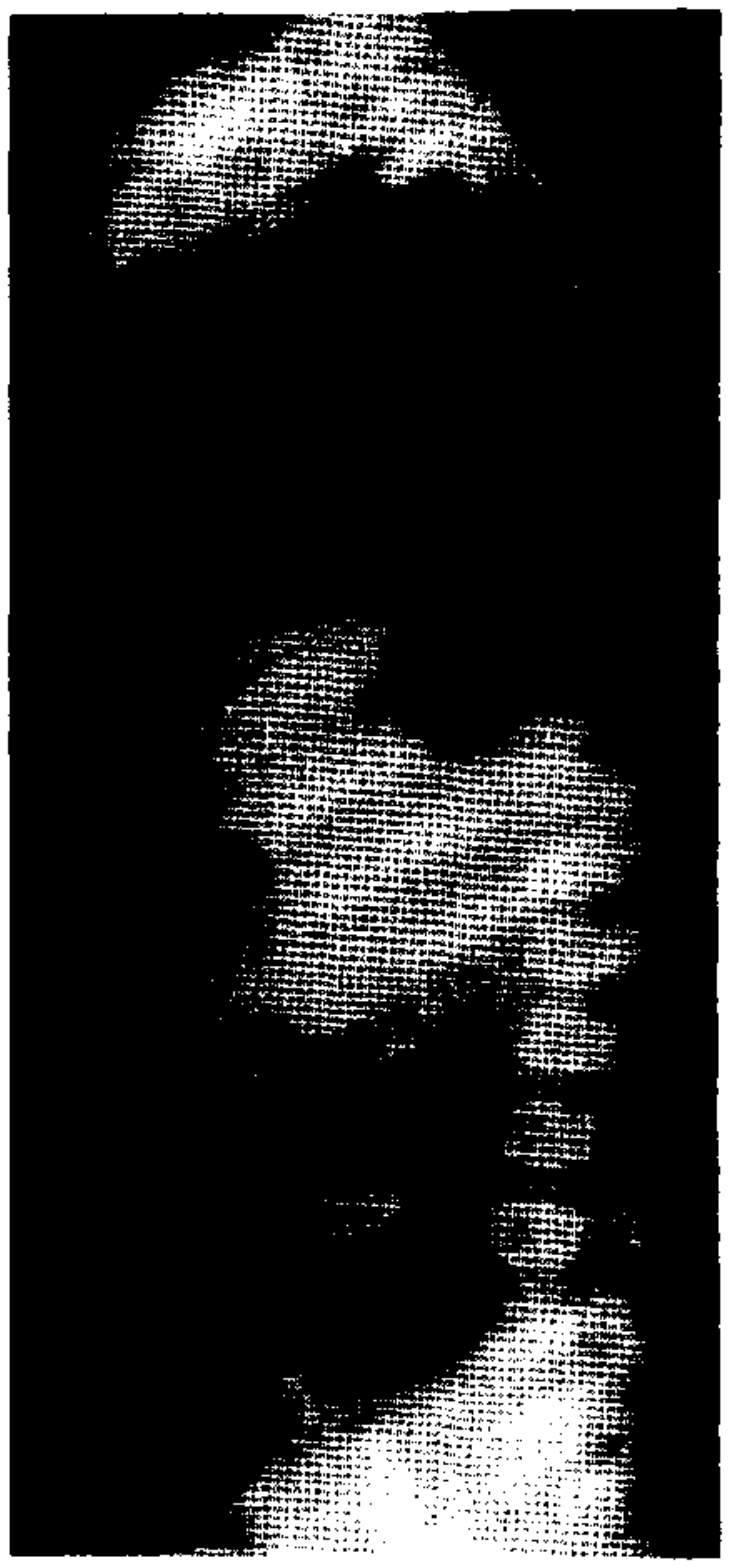

FIGURA I

RADJOGRAFIA LATERAL DE COLUMNA DEL PACIENTE A.M.R., QUE MLESTRA ULTIMAS VERTEBRAS DORSALES REDONDEADAS 
Casoll: V. C. P., 7 años. Paciente de sexo femenino producto de embarazo con infecciones urinarias a repetición e hipertension arterial materna. Parto por cosárea a los nueve meses. Score de apgar normal. Sin patologia en periudo de recién nacidn. Padres jorvenes no consanguineos. Padre portadur de hipr acusia unilateral y bronquitis erinica. La paciente presenta un desarrollo pondiestatural adecuado. Episudios de bronquitis, rinitis y otitis a repeticion Hesde los primeros años de vida. Retraso progresiv" de] desarrollo psicomotor consignado deste lis cuatro meses de vida, hipoactividad thipotonid: actualmente no habla ni regula esfínteres. Al examen fímico presentaba cabello grueso pajizo, facie tosca con cejas hiperpubladas, sinofrisis, labins gruesus y masenglesia, alteraciones dentarias, cuello cortn. Hipeareusia unilateral mixin. Tirax atmmbadus es1ertores pulmonares múltiples. Abdomen prominente con hernia umbilical y hepatomegalia. Dedos de manos y pies cortos y gruesos. Radiolígicamente presentaba engrosamiento óseo difuso especialmente en huesus del cráneu, silla turca normal. ruerpos vertebrales redondeados y costillas en espátula. Sin upacidades comeales ni alteraciones de retina. Screening de MPS positivo. MPS en orina de 24 horas aumentados en base a heparan sulfato. La aclividad de alfahexosaminidasa plasmática normal descartí un Sanfilippo B.

Caso III: A. C. P., 5 años ífigura 211. Paciente de sexi masculino hermano del caso II; producto de embarazo con hipertensión arterial severa materna e infecciones urinarias a repeticion. Parto y periodn de recitin nacido normales. Presenta episodios de bronquitis, otitis y renitis a repetícion desde los primeros años de vida. Ritmo de crecimiento pondopstatural adecuado. Desarrollo psicomotor normal hasta los 2 años de sida, luego retraso progresivo del área verbal y alteraciones de conducta con agresivjdad y trastornos del sueñor. Edad mental de 3 añis para 4 8/12 años de edad cronológica. Al examen físico destacaba cabello grueso, facie tosea con cejas hiperpohladas, sinofrisis y macroglosia: cuello corto. tórax simétrico, ron esterlores pulmonares múltiples. Abdomen glıbulosit con hernia umbilical y hepato-megalia. Dedos de manos y pies cortos y gruesus, con buena extensibilidad articular. Examen oftalmolígico normal. El exanen radiológico reveló retraso moderado de edad úsea, engrosamiento de huesus del cráneo, silla turca normal. El screening de MPS en orina fue positivo, con tipificacicin que reveló exceso de heparan sulfato. La determinación de actividad enzimática sérika fue normal para todas las alfahexusaminidasas presentes ell plasma. doscariando un Sanfilippo $\mathrm{B}$.

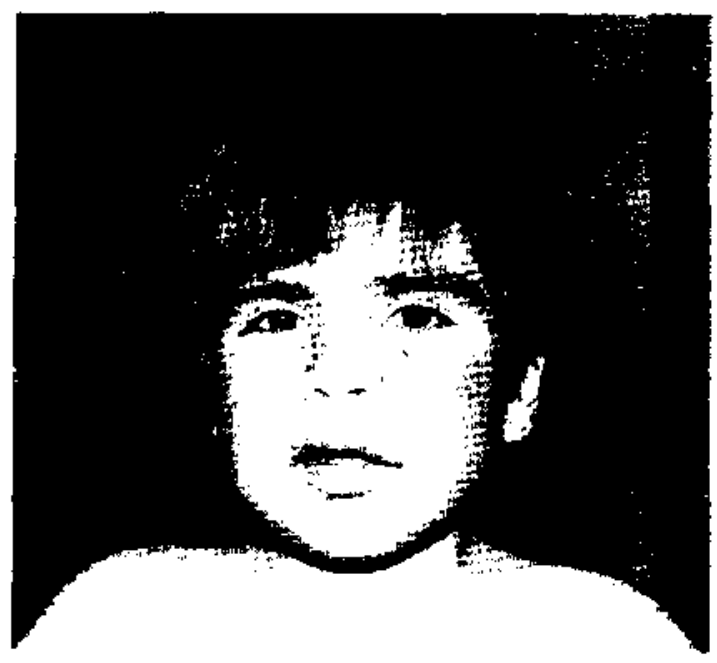

Fllik Ra H

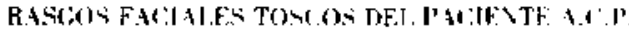

\section{DISCISION}

Los primerus test hiotpumicos para el diagmislionde las mucopolisacaridosio median el total do MPs ex. cretados en 24 horas. En los pacientes afectados los valores son superiores a $15 \mathrm{mg}$ siendo lo normal de 3 a $15 \mathrm{mg}$. En los heterocigotos estos valures sin int ermedios." Posteriormente se efectuis la tipifieacioin de MPS en orina, descrihiéndose modelos de excrecion para las diferentes tipos de mucopolisacaridosis, encontrándose aumento en orina de heparan y dermatan sulfato en el Hurler 4.5 so. Hunter y Scheie: sólo Heparan sulfato en el Sanfilippo, Keratan sulfato en el Morquio y Dermatan Sulfato en el Maroteaux Lamy y MPS tipo VII.

Fon los 3 casos presentados la tipificación de MPS en orina reveliu un exceso de heparan sulfato. In que certifics el diagnóstico de Sindrome de Sanfilippo. Para intentar incluir a eston pacientee en uno de las 3 tipos de Sanfilippo. se midiós actividad enzimática en plasma. lo cual permitis descartar un Sanfilippo B. pero no a un A o C. Para llegar al diag. nóstico de uno de estos dos tipos. seria necesario medir actividad enzimática en fibroblastos. pur no estar presentes en plasma o suero las enzimas deficitarias en estos 2 grupos. El estudio enzimático es necresario para el diagnóstico preciso de las mucopolisacaridosis y ha permitido comprobar variabilidad en el cuadro clínico de un mismo tipo de mucopolisacaridosis y signologia similar entre los diferentes tipos.

La determinación enzimática en células de lí- 
quido amniótico permite un diagnöstico prenatal preciso. ${ }^{11}, 15,16$ En recièn nacidos fallecidos con Síndrome Sanfilippo diagnosticados en la etapa prenatal se ha demostrado escaso o nulo compromiso del S.N.C. ${ }^{15}$ Conociendo que este Sindrome daña en forma severa y progresiva el S.N.C. resulta de gran interés el diagnóstico y tratamiento precoz de esta patología.

Se han descrito varios tratamientos para evitar acúrnulo de MPS en los diferentes órganos de pacientes. Las primeros intentos terapéuticos se hicieron con corticoides, vitamina A y dietas escorbutogénicas con resultados negativos en humanos. Posteriormente se ha intentado el reemplazo enzimático a través de transfusiones de sangre, plasma y leucocitos. publicándose resultados que difieren en su efectividad 17, 18 postulándose una posible formación de anticuerpus contra la enzima aportada en los casos de fracaso. 19, 2n

Los mejores resultados han sido obtenidos por Diferrante y Knudson 21 especialmente con infusiones de Leucocitos, con cambios espectaculares en el cuadro clínico que se mantienen casi por tres meses post transfusión a pesar que la excreción de MPS vuelve rápidamente a los valores alıos previos al tratamiento. En muctos casos considerados como fracaso. si bien no se ha obtenido mejoría en el asperto físico se ha logrado detener e incluso impedir el compromiso del S.N.C. ${ }^{22}$

En los casos presentados creemos que vale la pena intentar un tratamiento por la posibilidad de detener $o$ quizás mejorar el compromiso del S,N.C. que indudablemente ya existe pero que de no mediar tratamiento. este compromiso irá progresando rápidamente.

\section{RESUMEN}

Se presentan 3 casos clínicos de Sanfilippo o Mucopolisacaridosis tipo III, en los que se efectuó tipificación de MPS en orina que reveló un exceso de Heparan Sulfato y determinación enzimática en plasma que reveló normalidad en los niveles de AlfaHexosaminidasas, lo cual descartó un Sanfibpo B, siendo necesaria la determinación enzimática en fibroblastos para poder concluir si se trata de un Sanfilippo A o B. Se discute la importancia de la precisión diagnóstica con estas lécnicas de laboratorio en los diferentes tipos de Mucopolisacaridosis y especificamente el pronóstico y posibilidad terapéuticas del Síndrome de Sanfilippo.

\section{REFERENCIAS}

ILegum, C. P., Schorr, S. and Ferman, E. R. The Genetic Mucopolysaccharidoeses and Mucolipidoses. Advances in Pediatric. Vol. 22: 305, 1976. Review and Commert.

${ }^{2}$ Lorincz, A. E. The Mucopolysaceharitoses: Advances in understanding and treatment. Fediatric Annals 7: J04, 1978.

${ }^{3}$ Rich, C., Diferrante, N, and Archibuld, R. M. Acid mucopolysaccharides excretion in the urine of children. J. Lab. Clin. Med. 50: $686,195 ?$.

${ }^{4}$ Dorfman, A. and Larincz, A. E. Ocurrence of urinary acid mucopolyaccharides in the Hurler Syadrome. Proc. Nall. Acod. Sci. 43: 443, 1957.

$5_{\text {Linker, A., and Terry, K. D. Urinary acid mucopulysaceharides }}$ in the normal man and in Hurler's Syndrome. Proc. Soc. Exp. Biol. Med. 11: 743, 1963.

6Kaplan, D. Clasification of mucopolysaccharidoses besed on the pattern of mucopolysacchariduria. Amer. J. Med. 47: 721, 1969.

${ }^{7}$ McKusick, V. A. Heritable disorders of connective tissue. (3d. ed.: St. Lous: Ed. McKusick. V. A. The C. V. Musby Cumpany, 1966.

BKresse, H., Von Figura, $\boldsymbol{K}$, and Klein, $U$. A news biochemical subtype of the Sanfilippo Syadrome: Characterization of the storage material in cultured fibrohlast of Sanfilippo $C$ patients. European J. Biochem, 92: 333, 1978.

${ }^{9}$ Klein, U., Kresse, $H$. and Von Figura, K. Sanilippo Syndrome type C: Deficiency of acetylcos: Alfa-ejucosaminide-N-Acelyltransferase in skin fibroblart. Proc. Natl. Acad. Sei. 75: 51a5. 1978.

${ }^{10}$ Hall, C. W., Liebuers, 1, Di Notale P., Netsfeld, E. F. Enzymic diagnosia of the Genetic Mucopolysaccharide storage disordera. Methude Enzymol. 50: 439, 1978.

iIFrantantoni, J. C.. Neufeld, E. F., Unlendorf, B. W. and Jacobsun, C. B. Intreaterine diagnosis of the Hureles and Hunter Syndromes. N. England J. Med. 280: 686, 1969.

${ }^{12}$ Sly, W. S., Quinton, B.A., MC Alister, W. H. and Rimoin, D.L. Beta-Glucuronidase deficiency: Report of clinicgl, radiological and biochemical featires of a new mucopolysaceharidosis. J. Pediatr. 82: 249, 1973.

${ }^{13}$ Matalon, R. and Dorfman, A. Acid mucopolisaccharides in cultured human fibroblast. LANCET II: 838, 1969.

${ }^{14}$ Frantantoni, J. C, Hall, C. W. and Neufeld, E. F. Mutual corJection of the defect in cultured fibmblast. Science 162: 570 . 1968b.

${ }^{15}$ Greenwoud, R. S., Hillman, R. E., Alcala, H. and Sly, W. S. Sanfilippo. A syndrome in the fetus. Clinical Genetics 13: 241 . 1978.

${ }^{16}$ Harper, P. S. Laurence, K. M., Farkes, A., Wusteman, F. S.. Kree, H, Von Figura, K., Ferguson-Smith, M. A., Dunean, D. M., Logan, R. W., Hall, E., and Whiteman. P. Sanflippo A. Disease in the fetus. J. Med. Genel, 11: 123, 1974.

${ }^{17}$ Diferrante, $N$, Nichols, B, L, Donnelly, P. V., Neri, C., Hrgoveic, $\boldsymbol{R}$., and Berglund, $R$. K. Induced degradation of Gycosaminoglycans in Hurler's and Hunter's syndmence by plasma infusion. Proc. Natl. Acad. Sci. 68:303, 1971.

${ }^{10}$ Dekaban, A. S., Holden, K. F. and Constantopoulos, G. Effecte of fresh plasme or whole blood transfusions on patients with various types of mucopolysnccharidosig. Pediatrics 50: 688, 1972.

${ }^{19}$ Erickson, R. $\boldsymbol{P}_{1}$, Sondman, R., Robertson, $W_{.}$Van B., and Epstein, C. J.: Inefficacy of fresh frozen therapy of muco. polygactharidosis. Pedialrics 50: 693, 1972.

${ }^{20}$ Dean, $M . F$, Benson, $P$.F and Muir, $H$. The effects of infusions of normal plagena in Iree patients Sanfilippo gyndrome. Biochern. Soe. Tras18. 1: 284, 1974.

${ }^{21}$ Knudson, A. G., Jr, Diferrante, N, and Curtis, J. E. Effect of leukocite transfuejon in a child with type Il mucopolysaccharidosis. Proc. Natl. Açad. Sci, 68: 1738, 1971.

${ }^{22}$ Booth, L. W., and Nadler, H. C. Pleama infusions in a infant with Hurler's syndrorge. J. Pediatrics 82: 273, 1973. 